\title{
Reisen nach Brasilien auf den Spuren Humboldts
}

Alexander von Humboldt gelangte niemals nach Brasilien. Er hatte erfahren, dass die portugiesische Regierung eine Order erlassen hatte, ihn sofort festzunehmen, sobald er die Grenze zum portugiesischen Kolonialreich in Amerika überschreiten würde. $\mathrm{Zu}$ tief saß die Angst bei den portugiesischen Kolonialbehörden vor der Kolonialismuskritik des Preußen und den Folgen, welche diese kritische Sichtweise des Kolonialsystems auslösen könnte. Doch auch wenn Humboldt am Río Negro nur an die Grenzen, aber nicht ins Innere des portugiesischen Reiches kam, so wirkte seine Reise doch inspirierend für viele weitere Forschungsexpeditionen, die in der Folge tief ins Innere Brasiliens unternommen wurden.

Wir wollen an dieser Stelle zwei dieser Reisen kurz betrachten. Die reiseliterarische Schilderung der ersten Annäherung von Georg Heinrich Freiherr von Langsdorff an die Küsten Brasiliens steht von Beginn an im Zeichen der tropischen Fülle. So lesen wir im ersten Band seiner erstmals im Jahre 1812 in Frankfurt am Main erschienenen Bemerkungen auf einer Reise um die Welt in den Jahren 1803 bis $1807^{1}$ zunächst von einem am 18. Dezember 1803 durchgeführten Versuch - Humboldt befand sich damals noch auf seiner amerikanischen Forschungsreise -, sich der "Insel St. Catharina“" und damit der brasilianischen Küste zu nähern: „und schon bewillkommnten [sic] uns, in einer Entfernung von 60 bis 80 Seemeilen, mehrere Schmetterlinge, die wahrscheinlich durch einen starken Wind dem Lande entrissen waren.“3

Doch diese erste Begegnung mit ungeheuer großen und bunten, vielfarbigen Bewohnern der Neuen Welt muss aufgrund eines aufziehenden schweren Sturmes - der gleichsam für die andere, gefährliche Seite der Tropen, also die komplementär zur Fülle stehende Falle steht - zunächst abgebrochen werden, bevor dann am 21. Dezember die erste Berührung mit Brasilien erfolgt:

Kaum konnte ich, belebt von so manchen schönen Bildern meiner Einbildungskraft, die wiederkehrende Sonne erwarten, um die nahe paradiesische Gegend zu besuchen. Meine Ideen waren, ich gestehe es, groß und gespannt, dem ungeachtet übertraf nun, je mehr ich mich dem Lande näherte, die Wirklichkeit meine Erwartung.

1 Bemerkungen auf einer Reise um die Welt in den Jahren 1803 bis 1807 von G.H. von Langsdorff, Kaiserlich-Rissischer Hofrath, Ritter des St. Annen-Ordens zweiter Classe, Mitglied mehrerer Akademien und gelehrten Gesellschaften. Mit acht und zwanzig Kupfern und einem Musikblatt. 2 Bde. Frankfurt am Main: Im Verlag bey Friedrich Eilmans 1812.

2 Ebda., S. 27.

3 Ebda.

Ә Open Access. (c) 2020 Ottmar Ette, publiziert von De Gruyter. (cc)BY-NC-ND Dieses Werk ist lizenziert unter der Creative Commons Attribution-NonCommericial-NoDerivatives 4.0 Lizenz.

https://doi.org/10.1515/9783110650686-021 
Die an Farben, Größe, Bau und Verschiedenheit mannichfaltigen Blüthen, hauchten in die Atmosphäre eine Mischung von Wohlgeruch, die mit jedem Athemzug den Körper stärkte und das Gemüth erheiterte.

Große Schmetterlinge, die ich bisher nur als Seltenheiten in unsern europäischen Cabinetten sah, umflatterten viele, noch nie oder in unseren Gewächshäusern nur als Krüppel gesehene und hier in üppigem Wuchs blühende Prachtpflanzen.- Die goldblitzenden Colibri's umschwirrten die honigreichen Blumen der Bananenwälder und wiederhallender Gesang noch nie gehörter Vögel ertönte in den wasserreichen Thälern, und entzückte Herz und Ohr.-Dunkele, überschattete Wege schlängelten sich von einer friedlichen Hütte zur andern, und übertrafen an Schönheit und Anmuth, an Abwechslung und Einfalt jede noch so gekünstelte Anlage unserer europäischen Gärten.- Alles was ich um mich her sah, setzte mich durch seine Neuheit in Erstaunen und machte einen Eindruck, der sich nur fühlen aber nicht beschreiben lässt. $-^{4}$

Dies ist fürwahr eine prachtvolle Annäherung an die Neue Welt. In dieser kurzen, aber ästhetisch wie kulturtheoretisch wohldurchdachten Passage sind all jene Topoi versammelt, die seit der ersten Annäherung des Christoph Columbus an die Inselwelt der Antillen die Wahrnehmungs- und Darstellungsmuster von Europäern prägen, welche die unterschiedlichsten Phänomene der für sie 'neuen' Welt im Zeichen des Reichtums und der Überfülle erstmals wahrnehmen. Alles ist neu, alles ist bezaubernd, alles ist betörend.

In einer Art Überbietungsstrategie setzt sich der amerikanische locus amoenus an die Stelle des weitaus kargeren europäischen 'Originals', ohne freilich im Geringsten die Darstellungsmodi der abendländischen Antike zu verlassen. Denn keines der Ingredienzien abendländischer Rhetorik fehlt. Reichtum und Exuberanz prägen alles, was sich den Sinnen des ankommenden Europäers darbietet. Es ist zugleich ein Diskurs der amerikanischen Fülle (und hier nur kurz angedeuteten tropischen Falle), deutlich nach jenem anderen, insbesondere das letzte Drittel des 18. Jahrhunderts dominierenden europäischen Diskurs angesiedelt, der unter Rückgriff auf Buffon oder de Pauw Amerika im Zeichen der Unterlegenheit und der Schwäche sah. Wir haben dieses Thema bereits in unserer Vorlesung bei den Überlegungen zur Berliner Debatte um die Neue Welt ausführlich aufgerufen.

Der Leiter dieser Expedition nach Brasilien war kein Unbekannter. Als Teilnehmer der ersten russischen Weltumsegelung, die unter dem Befehl von Adam Johann von Krusenstern durchgeführt wurde, hatte sich Langsdorff - wie er in seinem auf St. Petersburg, den 12. Juni 1811 datierten Vorwort zu seinen Bemerkungen auf einer Reise um die Welt ausführte - ,als Arzt und Naturforscher“5 erstmals der amerikanischen Hemisphäre zugewandt. Er partizipierte

4 Ebda., S. 29.

5 Ebda., S. xix. 
damit an einer Unternehmung, wie sie charakteristisch war für die zweite Phase beschleunigter Globalisierung: Weltumsegelungen also, wie sie auf französischer Seite Bougainville und „der unsterbliche“6 Lapérouse oder auf britischer Seite James Cook - mit Georg Forster 'im Gepäck' - durchgeführt hatten.

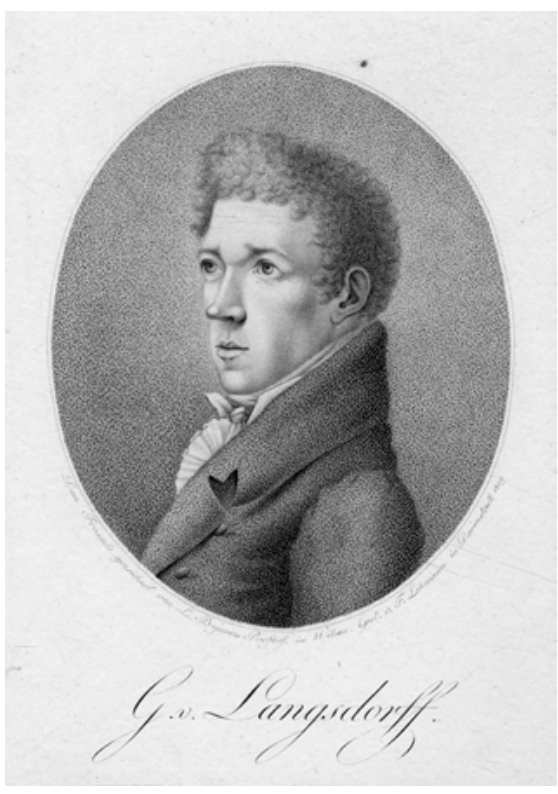

Abb. 76: Georg Heinrich von Langsdorff (Wöllstein in Rheinhessen, 1774 - Freiburg im Breisgau, 1852).

Das Russische Reich war auf diesem Gebiet ein Nachzügler; und so verwundert es nicht, dass lange Jahrzehnte die paradigmatischen Weltumsegelungen der Franzosen und Engländer von jener der Russen trennten, die ihrerseits ein ausgeprägtes Interesse insbesondere an der Erforschung der Küsten des Pazifik und des russischen Amerika besaßen. Nicht zuletzt auch die Erforschung der Halbinsel Kamtschatka lag im Visier der russischen Weltumsegelungen. Noch über ein Jahrzehnt später sollte Adelbert von Chamisso an einer weiteren russischen Weltumsegelung in den Jahren 1815 bis 1819 teilnehmen, die unter dem Kommando Otto von Kotzebues stand. Doch werden wir uns an dieser Stelle nicht mit dem faszinierenden Reisebericht Chamissos beschäftigen, der auf der

6 Ebda., S. 26. 
russischen Brigg 'Rurik' insbesondere den Südpazifik und Hawaii, aber auch die Aleuten und die Halbinsel Kamtschatka erforschte. ${ }^{7}$

Doch zurück zu Langsdorffs Unternehmung. Im Vergleich mit den genannten Weltumsegelungen ist es aufschlussreich zu konstatieren, dass in der Figur von Georg Heinrich Freiherr von Langsdorff ein grundlegender Paradigmenwechsel aufscheint, der sich noch im Verlauf dieser zweiten, im Zeichen der Führungsmächte England und Frankreich stehenden Beschleunigungsphase der europäischen Globalisierung vollzog: der Wechsel von der Entdeckungsreise (sei es in Form von Seereisen zu bestimmten Küstenstrichen, sei es in Form spektakulärer Weltumsegelungen) zur Forschungsreise, wobei die erstere stets nur die Küstenbereiche berührte, die zweite hingegen auf eine Erforschung gerade auch der Binnenräume der Kontinente abzielte. Dies war ein folgenreicher Paradigmenwechsel, für den gerade auch Humboldts Reiseunternehmung stellvertretend stehen mag. Denn dieser hatte in den ausgehenden neunziger Jahren noch darauf gehofft, sich einer weiteren französischen Weltumsegelung unter Kapitän Baudin anzuschließen, bevor er sich - durchaus mit hohem Risiko und auf eigene Kosten - im Juni 1799 zusammen mit Aimé Bonpland auf seine eigene Forschungsreise in die amerikanischen Tropen begab. Diese führte ihn wiederholt auch ins Innere des amerikanischen Kontinents.

Zwei Jahrzehnte nach der erwähnten Krusenstern'schen Weltumsegelung erfüllte die von 1824 bis 1828 durchgeführte Langsdorff'sche Expedition - wiederum in russischem Auftrag - alle Kriterien jener nun vermehrt angestrebten Erforschung des Landesinneren, die nunmehr im Zentrum der europäischen Expansionsbemühungen - seien sie vorwiegend wissenschaftlicher oder politischer beziehungsweise ökonomischer Ausrichtung - stand. Langsdorff selbst hatte die wissenschaftliche Notwendigkeit eines langfristigen Aufenthalts in Brasilien bereits in seinem Reisebericht bezüglich der Insel Santa Catalina festgehalten. In einer Art Vorwegnahme seines späteren Lebens merkte er im Angesicht der Überfülle von Naturphänomenen in einer epistemologisch wie autobiographisch nicht unwichtigen Fußnote an, dass man hier eines Botanikers bedürfe, „der sich nicht Tage und Wochen, sondern Jahre lang hier

\footnotetext{
7 Vgl. zu den Verbindungen dieser Reise gerade auch zu den Unternehmungen von Georg Forster und Alexander von Humboldt Ette, Ottmar: Welterleben / Weiterleben. Zur Vektopie bei Georg Forster, Alexander von Humboldt und Adelbert von Chamisso. In: Drews, Julian / Ette, Ottmar / Kraft, Tobias / Schneider-Kempf, Barbara / Weber, Jutta (Hg.): Forster - Humboldt - Chamisso. Weltreisende im Spannungsfeld der Kulturen. Mit 44 Abbildungen. Göttingen: V\&R unipress 2017, S. 383-427.
} 
aufhalten muss“, könne dieser Forscher doch nur so „durch die Entdeckung einer Menge neuer genera und species an Pflanzen belohnt werden.“ ${ }^{* 8}$

Georg Heinrich Freiherr von Langsdorff verkörpert als Teilnehmer wie als der spätere Leiter einer Entdeckungs- wie einer Forschungsreise damit einen paradigmatischen Wechsel, der in seinem Falle gerade angesichts der Schwierigkeiten, auf die seine Expedition stieß - zahlreiche Dokumente belegen die internen Spannungen zwischen den einzelnen Mitgliedern seiner Expedition ${ }^{9}$-, auch das Oszillieren zwischen Fülle und Falle miteinschloss. Denn das, was sich zunächst den Sinnen des europäischen Reisenden als Fülle darbot, konnte sich schon rasch und jederzeit in eine gefährliche Falle verwandeln. ${ }^{10}$ Dass sich der noch junge Reisemaler Johann Moritz Rugendas seinerseits der Falle, welche für ihn die Langsdorff-Expedition darstellte, zu entziehen vermochte, wirft ein bezeichnendes Licht auf die große Bedeutung, welche dem Zusammenleben, der Konvivenz, für das Überleben und den wissenschaftlichen wie künstlerischen Ertrag jedweder Forschungsreise zukommt. ${ }^{11}$ Der autoritäre Führungsstil Langsdorffs war offenkundig den wissenschaftlichen Ergebnissen nicht immer zuträglich. Doch können wir an dieser Stelle dem Spannungsverhältnis zwischen ihm und dem hochtalentierten Maler Rugendas (der zu jenen Malern zählte, die später auf den Spuren Humboldts und mit seiner Idee von Landschaftsmalerei im Kopf weite Bereiche des späteren Lateinamerika bereisten) nicht nachgehen.

Der Bericht von Langsdorff ist in vielerlei Hinsicht aufschlussreich, liefert er nicht zuletzt doch ebenso wie Antoine-Joseph Pernety ein eindrucksvolles Bild von einer Äquatortaufe, diesmal freilich an Bord eines russischen Schiffes. ${ }^{12}$ Auch die umfangreichen Ergebnisse Langsdorffs können sich sehen lassen, führte ihn doch seine Reise bis tief hinein ins brasilianische Hinterland und selbst bis in jenen 'Wilden Westen' Brasiliens, der zugleich auch eine der klimatisch anspruchsvollsten Zonen des Landes rund um Cuiabá darstellt. Anders als Pernety verfiel Georg Heinrich Freiherr von Langsdorff nicht in den Fehler einer

8 Langsdorff: Bemerkungen auf einer Reise um die Welt in den Jahren 1803 bis 1807, S. 49.

9 Vgl. hierzu die wichtige Zusammenstellung von Costa, Maria de Fátima / Diener, Pablo (Hg.): Viajando nos Bastidores: Documentos de Viagem da Expediçao Langsdorff. Cuiabá: Ministério da Educaçao e do Desporto 1995.

10 Zur historischen Dimension des Wechselspiels von Fülle und Falle vgl. insbes. Kapitel III in Ette, Ottmar: Konvivenz. Literatur und Leben nach dem Paradies. Berlin: Kulturverlag Kadmos 2012, S. 102-146.

$11 \mathrm{Zu}$ den Dokumenten der Zerrüttung zwischen Langsdorff und Rugendas in Brasilien vgl. Costa, Maria de Fátima / Diener, Pablo: Entorno dos documentos. In (dies., Hg.): Viajando nos Bastidores: Documentos de Viagem da Expediçao Langsdorff, S. 20-25.

12 Vgl. hierzu Langsdorffs Darstellung in seinen Bemerkungen auf einer Reise um die Welt in den Jahren 1803 bis 1807, S. $22 \mathrm{f}$. 
idealisierenden Darstellung. Seine Arbeitsergebnisse waren durch ein angestrebtes Höchstmaß an faktentreuer Beschreibung charakterisiert.

So zeigt er sich etwa im fünften wie im sechsten Kapitel seines Reiseberichts stets darum bemüht, die gesellschaftlichen Verhältnisse wie auch die Religion, zugleich aber auch die Kunstfertigkeiten und künstlerischen Dimensionen des Lebens der von der Krusenstern'schen Expedition besuchten Bewohner der Marquesas- und der Washington-Inseln so präzise als irgend möglich darzustellen. Gerade der Kunst der Tätowierung wendet er sich im Kontext ihrer sozialhierarchischen Bedeutung ausführlich zu. ${ }^{13}$ Langsdorffs autoritärer, herrschaftlicher Führungsstil stand mit seiner eher zurückhaltenden Beschreibung vieler Fakten und Dokumente in einem eigenartigen Kontrast.

Selbst die bei europäischen Reisenden oftmals höchst voreingenommene Reflexion der Anthropophagie wird von Langsdorff unter der Überschrift 'Nahrung ${ }^{14}$ so abgehandelt, dass in keinerlei Weise die disqualifizierenden und aus dem Menschengeschlecht exkludierenden Äußerungen de Pauws in den Horizont seiner Betrachtungen rücken. Zugleich enthält sich Langsdorff jeglichen Versuches, das Zusammenleben der Bewohner der Marquesas zu idealisieren, auch wenn sicherlich eine grundsätzliche Exotisierung aller Lebensverhältnisse in seinem Reisebericht festzustellen ist. Eine gewisse Ausnahme bilden freilich - die Eingangspassage hatte uns hierfür bereits ein Beispiel gegeben - all jene Passagen in Langsdorffs Bericht, die sich auf Brasilien als tropischen locus amoenus beziehen. Sie tropikalisieren dann freilich alles und staffieren diese paradiesartig überhöhten Passagen mit nicht wenigen idyllischen Zügen aus; denn nicht selten schildert uns Langsdorff immer wieder Teile Brasiliens als eine „paradiesische Gegend“. ${ }^{15}$ Offenkundig war der Reiz, dies für ein europäisches Lesepublikum zu tun, zu groß oder auch der Erwartungsdruck zu hoch.

Aber insgesamt steht dann Langsdorffs Reisebericht über die eigene russische Expedition von 1824 bis 1828 doch auf der Seite jener empirischen Datenerfassung und damit jener präzise darstellenden Reisenden, welche die wissenschaftliche Reiseliteratur im neuen Jahrhundert überwiegend prägte und einer nicht faktenorientierten Darstellung durch Daheimgebliebene den Boden entzog. Er verbreiterte die Grundlage des Wissens über die Neue Welt und speziell über Brasilien auf nachhaltige Weise. Insofern spielt in seinen Schriften noch immer ein Echo der Berliner Debatte um die Neue Welt eine gewisse hörbare Rolle. Schreibt er sich auch mit seiner nur durch ein glückliches Zusammenspiel von Zufällen zustande gekommenen

13 Vgl. ebda., S. 99-108.

14 Ebda., S. 99.

15 Vgl. ebda., S. 29. 
Teilnahme an der großen russischen Weltumsegelung deutlich auf der Seite der philosophes voyageurs ein und richtet sich seine eigene Expedition auch dezidiert am neuen empirisch fundierten Forschungsparadigma aus, so verdankt gerade seine Auseinandersetzung mit unterschiedlichsten indigenen Kulturen doch noch manches den Einschätzungen eines de Pauw und dessen Anhängern.

Die Liebeserklärung, die Langsdorff bereits in seinem Reisebericht an Brasilien richtet, ist freilich weit mehr als die emphatische Äußerung eines subjektiven Gefühls; vielmehr schreibt der Forscher, dass er mit der Fortsetzung der russischen Weltumsegelung „das schönste und reichste Land der Erde verlassen" ${ }^{\text {16 }}$ habe. Und er fügt noch einen Satz hinzu, in dem sich bereits die Konturen eines Lebensprojekts abzuzeichnen scheinen: „Die Rückerinnerung an meinen Aufenthalt in Brasilien, wird mir zeitlebens unvergesslich bleiben! ${ }^{\text {‘17 }}$ Umgekehrt ist in Brasilien - wie in jüngster Zeit eine Tagung zu Langsdorffs Reise zeigt - die Erinnerung an seine Expedition und seinen Reisebericht nicht verloren gegangen, sondern lebendig geblieben.

Aber noch wesentlich lebendiger und stärker ist in Brasilien freilich die Erinnerung an eine Reise zweier bayerischer Naturforscher, die größte Teile des Landes bereist und beschrieben haben und so etwas wie die wissenschaftlichen Entdecker Brasiliens geworden sind. Es ist die Reise der Forscher Johann Baptist von Spix und Carl Friedrich Philipp von Martius, die 1817 im Auftrag der Königlich-Bayerischen Akademie der Wissenschaften nach Brasilien kamen und drei lange und ereignisreiche Jahre lang im Lande blieben.

Sie waren Teil des Gefolges der Tochter des österreichischen Kaisers, Leopoldina, die als Ehefrau des künftigen portugiesischen Kaisers Dom Pedro I. nach Rio de Janeiro zu ihrer Vermählung reiste. Doch bald schon, nach wenigen Monaten, waren die Verpflichtungen der Wissenschaftler bei Hofe vorüber und ihre mehr als dreijährige brasilianische Reise begann. Es ist wohl die Reise, an welche sich die Brasilianer bis heute am eindrücklichsten erinnern und die bis in die neueste Zeit auch auf ein großes Echo innerhalb der brasilianischen Wissenschaft gestoßen ist. ${ }^{18}$ Denn die Veröffentlichungen von Spix und Martius waren ohne jeden Zweifel das Beste, das über Brasilien im 19. Jahrhundert veröffentlicht wurde. Zumindest aus naturhistorischer Sicht. Denn in der Anthropologie gab es, wie wir noch sehen werden, ein mit der hier skizzierten Geschichte sehr eng zusammenhängendes Problem.

16 Ebda., S. 67.

17 Ebda.

18 So hat beispielsweise das von Eckhard E. Kupfer, Daniela Rothfuss und Birgit Fouquet herausgegebene Martius-Staden-Jahrbuch die Nummer 62 (2018) als zweisprachige Ausgabe fast vollständig der Reise von Spix und Martius gewidmet. 


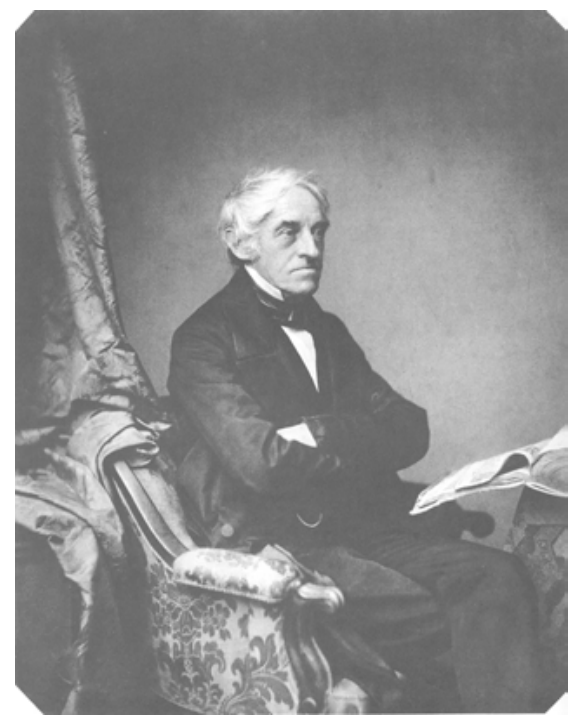

Abb. 77: Carl Friedrich Philipp von Martius (Erlangen, 1794 - München, 1868).

Ich spielte gerade mit dem Entdeckungsbegriff mit Blick auf Brasilien. Das ist natürlich mit einem Augenzwinkern zu verstehen, doch man darf sich die Kenntnisse der Kolonialbehörden über dieses riesige Land als völlig ungefähr und rudimentär vorstellen. In den großen Städten an der Küste oder in Küstennähe wusste man wenig vom Landesinneren, wenn dort nicht Gold- oder andere Minen zu finden waren. Man nahm sogar innere Gebirgszüge dort an, wo der Amazonas sein gewaltiges Becken entwickelt hatte, sah die Grenzen des Landes im Ungefähren und hatte nur diffuse Vorstellungen von all jenen gewaltigen Gebieten, die im Schatten der kolonialen Ausbeutung geblieben waren. Noch im 19. Jahrhundert war Brasilien den Portugiesen, aber auch den einheimischen Eliten in weitem Maße unbekannt.

Dies sollte sich mit der Reise von Spix und Martius grundlegend ändern. Insgesamt blieben die beiden Forscher zunächst einmal fast sechs Monate lang in Rio de Janeiro, wo sich der portugiesische Königshof befand, um sich zu akklimatisieren, die Umgebung an der Küste und im unmittelbaren Küstengebirge $\mathrm{zu}$ inspizieren und erste botanische Expeditionen und Versuche anzustellen. Hierbei kam es insbesondere zur Untersuchung der sogenannten 'Mata Atlântica', des Küstenregenwaldes, der in Brasilien am Gebirgsanstieg ins Landesinnere eine besondere Rolle spielt. Die beiden Forscher waren von der sich ihnen darbietenden Tropenwelt begeistert. Erst Ende des Jahres gingen beide dann in Begleitung des berühmten Malers Thomas Ender, der später auch Humboldt portraitieren sollte, nach São Paulo und damit erstmals ins Landesinnere, 


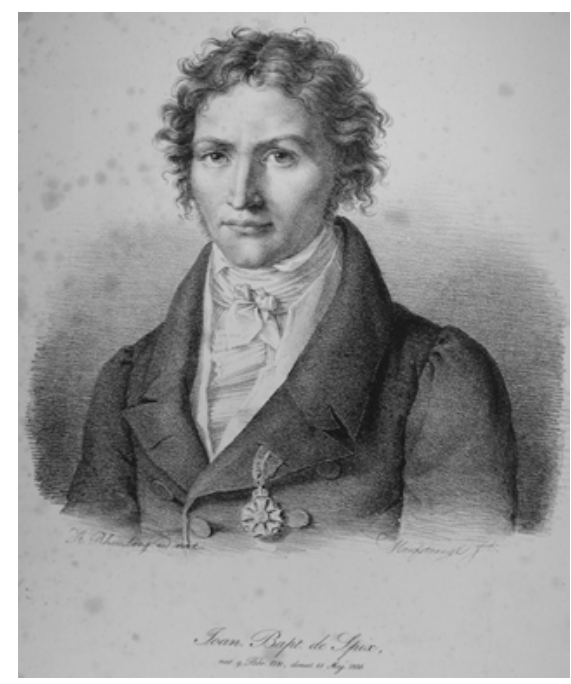

Abb. 78: Johann Baptist von Spix (Höchstadt an der Aisch, 1781 - München, 1826).

welches von Anfang an das eigentliche Ziel ihrer Expedition war. Es begann eine Reise mit ungeheuren Mühen und Strapazen, aber zugleich auch mit Ergebnissen von fundamentaler Bedeutung für die wissenschaftliche Erforschung Brasiliens.

Denn was nun folgte, war ein Kraftakt forscherischer Anstrengung, insofern große Teile von Minas Gerais, des Sertão und Inner-Brasiliens mühselig durchquert wurden und Städte wie Bahia dabei als Stützpunkte, aber auch als weitere Untersuchungsgegenstände hinzukamen. Tausende von Kilometern legten die beiden Forscher zurück und lernten weite Teile des tropischen Landes kennen.

Aufschlussreich ist, dass in einer neueren Studie etwa die Frage der Nachhaltigkeit an die Arbeiten von Spix und Martius herangetragen und letztlich positiv beantwortet werden konnte. ${ }^{19}$ Immer wieder treten der Fortschrittsgedanke und vielleicht mehr noch der Gedanke an die Zukunft des Landes neben alle Landschaftsbeschreibungen von majestätischen Wäldern und die bange Ahnung, dass Brasilien einer vielleicht großen, vielleicht aber auch ungewissen Zukunft entgegengehen könnte:

19 Vgl. Paca, Sérgio A.: Reise in Brasilien und die aktuellen Ziele der nachhaltigen Entwicklung. In: Martius-Stademn-Jahrbuch (São Leopoldo) 62 (2018), S. 69-82. 
Was wohl das vierte Jahrhundert über ein Land heraufführen werde, das blos in den drei verflossenen schon alle Richtungen und Bildungsstufen aufzunehmen vermochte, durch welche der Genius der Menschheit die alte Welt während Jahrtausenden hindurchgeleitet hat? ${ }^{20}$

In diesen kurzen Sätzen liegt für Spix und Martius das ganze Geheimnis weiter Landstriche, die binnen kurzer historischer Zeit die gesamte Menschheitsentwicklung durchlaufen mussten und künftig auch noch durchlaufen würden. Wie aber waren die physischen und kulturellen Voraussetzungen für einen solchen Weg menschheitsgeschichtlicher Beschleunigung beschaffen? Denn es war klar und deutlich, dass weite Teile des Landes noch in keiner Weise dem Entwicklungsstand etwa West- oder Mitteleuropas entsprachen.

Die Portugiesen waren an einer kolonialen Ausbeutung von Ressourcen, nicht aber an einer guten Entwicklung des gesamten Landes interessiert. Aus kolonialistischer Sorge war noch die gesamte universitäre Ausbildung - anders als in den ehemaligen spanischen Kolonien Amerikas - in Portugal, im heimischen Coimbra, angesiedelt, so dass auch auf diesem für die Entwicklung der Wissenschaften zentralen Gebiet immenser Nachholbedarf bestand. So war die Frage, wie sich das künftige Brasilien dereinst entwickeln würde, für die beiden ausländischen Forscher eine zentrale Herausforderung. Es waren immer wieder solche Vorstellungen, die für Spix und Martius Anlass gaben zu tieferen philosophischen Einlassungen über die Zukunft jenes Landes, das sie so intensiv bereisten.

Und dann gab es neben allen anderen Problemen einer ökonomischen, industriellen, wissenschaftlichen und sozialen Entwicklung ja auch noch den Komplex - für Spix und Martius die schwere Bürde - der in Amerika einheimischen Kulturen. Martius und Spix wagten sich auf ihrer Reise auch in weite Teile des Amazonas-Tieflandes hinein und kamen oftmals in Kontakt mit der dort ansässigen und häufig mit den Weißen nicht vertrauten indigenen Bevölkerung. Dabei ist auffällig, dass diesen bayerischen Wissenschaftlern so gar nichts mehr anhaftet von Jean-Jacques Rousseaus Enthusiasmus für den homme naturel, der in großer Einfachheit, aber grundlegender Einheit mit der Natur lebe und einen höheren moralischen Seinsgrund als die von der Zivilisation verdorbenen und degradierten Europäer besitze.

Die Sichtweise der beiden Bayern sah anders aus. Die Bilanz im dritten Band des von Carl Friedrich Philipp von Martius verfassten Reiseberichts fiel in

20 Spix, Johann Baptist von / Martius, Carl Friedrich Philipp von: Reise in Brasilien. 3 Bände. Stuttgart: Brockhaus 1980, Bd. 2, S. 644. Vgl. hierzu auch die schöne Überblicksstudie von Macknow Lisboa, Karen: Die Reise von Spix und Martius im 19. Jahrhundert nach Brasilien. In: Martius-Staden-Jahrbuch (São Leopoldo) 62 (2018), S. 6-19. 
vielerlei Hinsicht sehr negativ aus. Er glaubte, 'den Indianer' kurz und knapp charakterisieren zu können:

Das Band der Liebe ist schlaff; statt Zärtlichkeit, Brunst; statt Neigung, Bedürfnis; [. . .] das nackte Weib, Sklavin; [...] die Ehe, ein nach Laune wechselndes Konkubinat; des Hausvaters Sorge, sein Magen; sein Zeitvertreib, Völlerei und dumpfes Nichtstun; [...] der Weiber Schaffen, blind und ohne Ziel; [...] die Erziehung, äffische Spielerei der Mutter und blinde Sorglosigkeit des Vaters; [...] statt Recht, die Stimme des Egoismus [...] - So ist und lebt der Unmensch dieser Wildnis. Auf der rohesten Stufe der Menschheit [. . . ${ }^{21}$

Das Urteil von Martius fällt also verheerend aus. Gewiss, er scheint sich im Alter wohl noch etwas besonnen zu haben und hat wohl sein Bild der 'Wilden' in einem Roman noch etwas zum Positiven hin korrigiert. ${ }^{22}$ Aber wir sehen hier ohne jeden Zweifel, wie stark das Indianerbild (oder Amerikanerbild) von Cornelius de Pauw bei deutschen Wissenschaftlern fortwirkte und wie sehr es ihre Sichtweise der indigenen Bevölkerung beherrschte. Es war ganz offensichtlich schwierig, aus diesen europäischen Überzeugungen und Stereotypen herauszukommen und ein eigenständiges, differenziertes Bild indigener Kulturen zu entwickeln. Bei Alexander von Humboldt ist dies freilich $\mathrm{zu}$ beobachten, zeigen seine Amerikanischen Reisetagebücher doch zu Beginn bisweilen ein ähnlich von einem starken europäischen Überlegenheitsgefühl geprägtes Bild, das er dann aber im Verlauf des Fortgangs seiner Reise immer mehr korrigierte und modifizierte, bis daraus ein reges Interesse an den indigenen Kulturen und Sprachen erwuchs. Das Interesse von Martius und Spix an der indigenen Bevölkerung freilich war nur das europäischer Forscher gegenüber naturhistorischen Gegenständen, die sie letztlich auch sehr verallgemeinernd und abwertend beschrieben. Die obigen Formulierungen deuten unverkennbar an, wie sehr die indigene Bevölkerung von der europäischen Wissenschaft geradezu aus dem Menschengeschlecht ausgebürgert wurde. Das in Europa verbreitete Rassedenken war auf dem Weg zu einem pseudo-wissenschaftlich begründeten Rassismus. Doch vieles im Reisebericht von Spix und Martius ist auf festeren Grund gebaut und unterliegt nicht mitgebrachten Vorurteilen europäischer Provenienz.

Auch in anderer Hinsicht sind ihre Schriften für ihre Zeit typisch. So verteidigten beide auch ganz deutlich die Vorstellung, dass Naturforscher in der Lage sein müssten, die Natur zu spüren, um sie danach erst konkret zu beschreiben und kategorisierend einteilen zu können. Dies ist zweifellos ein Aspekt einer romantischen Naturforschung, wie sie sich nicht nur in Deutschland weit verbreitet

21 Ebda., Bd. 3, S. 1268.

22 Vgl. hierzu Macknow Lisboa, Karen: Die Reise von Spix und Martius im 19. Jahrhundert nach Brasilien, S. $17 \mathrm{f}$. 
hatte. Hierfür sind unsere bayerischen Forscher gute, fast modellhafte Beispiele. Das schreiben im Zeichen einer romantischen Naturerfahrung war zweifellos auch ein Schreiben in einer europäischen Moderne, die längst auch nach Amerika überzuschwappen begann.

So schwingt in den von Spix und Martius verfassten Beschreibungen der Natur immer wieder ein etwas euphorisierendes, in jedem Falle bewunderndes Bild der tropischen Natur mit, wie wir es im Grunde seit Columbus im europäischen Diskurs kennengelernt hatten. Es ist auch jener Ton, den wir bereits bei Langsdorff gehört hatten, eine Bewunderung für das Majestätische der Natur, für ihr fremdländisches Aussehen, ihre wunderbare Fülle. Alle Gemeinplätze europäischer Provenienz versammeln sich hier. Diese Passagen sind gerade auch in Brasilien noch heute recht populär und bezeugen ein Bild von der Natur, wie es dem klassischen europäischen Topos des locus amoenus in einem tropikalisierten Kontext entspricht. Ich muss sie daher an dieser Stelle nicht nochmals zitieren, wir sind derlei Formulierungen in unserer Vorlesung häufig genug begegnet. Die europäischen Versatzstücke des Diskurses haben sich also nicht grundlegend geändert: Wir stoßen auch heute noch etwa in der Werbung für Fernreisen immer wieder auf sie.

Zugleich fasziniert aber, mit welcher Akribie und Präzision sich die bayerischen Wissenschaftler in die ungeheure Fülle der Naturerscheinungen einarbeiten und die unterschiedlichsten Aspekte und Phänomene dieser tropischen Natur, in Flora und Fauna, genauestens untersuchen. Der wissenschaftliche Reisebericht gerät hier rasch zu einem in romantischer Naturlyrik schwelgenden Bericht, der seine literarischen Quellen kaum verleugnen kann. Doch er geht zugleich über in die genaueste Kartierung und Klassifizierung, die man der Natur eines Landes, das man bereist, angedeihen lassen kann. Übrigens auch in einem ganz kartographischen Sinne, wurde die Amazonas-Karte des Jesuitenpaters Samuel Fritz doch von dem Franzosen La Condamine (vgl. Abb. 62: Amazonas-Karte in La Condamines Relation abrégée d'un voyage fait dans l'intérieur de l'Amérique méridionale) - wie wir sahen - umgearbeitet und vervollständigt, dann aber von Spix und Martius weiterentwickelt und perfektioniert.

Eines ist gewiss: Das Bild von Brasilien war ein anderes vor und nach der Reise von Spix und Martius - ebenso in Europa wie in Brasilien selbst.

Nach dem frühen Tode von Spix fiel die ganze Last der Auswertung der gemeinsamen Reise auf Martius, der damit auch in der Tat bis zu seinem Lebensende beschäftigt war. Noch immer beherbergen sowohl die Münchener Staatsbibliothek als auch die Bayerische Akademie der Wissenschaften den wissenschaftlichen Nachlass der beiden Forscher, der noch heute eine wichtige Fundgrube darstellt für historische Forschungen vor Ort. Hier lässt sich in allen 
Details die Entwicklung eines komplexen Bildes insbesondere der Naturgeschichte Brasiliens nachvollziehen: Bilder und Entwürfe, die an Präzision nur wenig zu wünschen übrig lassen.

Mit ihrer Reise in Brasilien haben Spix und Martius in gewisser Weise Brasilien nicht nur vorgefunden und beschrieben, sondern vielleicht mehr noch erfunden und auf einen Weg der zunehmenden Entdeckung gerade auch durch die einheimischen Eliten gebracht. Dies ist ein Weg, der bis heute noch nicht abgeschlossen ist und noch viele Anstrengungen der Wissenschaft erfordert. Aber auch einer wissenschaftlichen Rezeptivität in der brasilianischen Gesellschaft, ein Aspekt, der gerade unter der neuen brasilianischen Regierung mehr als deutlich in Frage steht. Wie wird sich Brasilien im 21. Jahrhundert entwickeln? Es ist fast überflüssig zu sagen, dass schon die bayerischen Wissenschaftler vor einer großflächigen $\mathrm{Ab}$ holzung des Amazonas-Regenwaldes warnten. Es sollte noch mehr als ein Jahrhundert vergehen, bevor diese Warnungen ernst genommen wurden. Mit der Umsetzung in ein politisches Handeln hapert es angesichts der Profitgier weniger Unternehmen und Großfamilien freilich noch heute. Die Abholzung der brasilianischen Regenwälder geht heute wieder verstärkt weiter - und mit ihr die Missachtung der Lebensansprüche indigener Kulturen. 